\title{
Impact of Medium Cut-Off Dialyzers on Patient-Reported Outcomes: COREXH Registry
}

\author{
Juan Carlos Alarcon ${ }^{a}$ Alfonso Bunch ${ }^{a}$ Freddy Ardila $^{b}$ Eduardo Zuñigab \\ Jasmin I. Vesga ${ }^{b} \quad$ Angela Rivera $^{c}$ Ricardo Sánchez ${ }^{d}$ Rafael Mauricio Sanabria ${ }^{a}$ \\ on behalf of the Colombian Registry of Expanded Hemodialysis Investigators

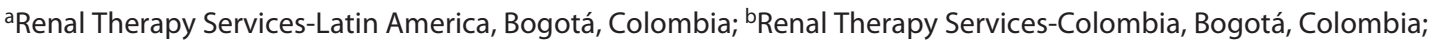 \\ 'Baxter Healthcare Corporation, Deerfield, IL, USA; ' Universidad Nacional de Colombia, Bogotá, Colombia
}

\section{Keywords}

Hemodialysis · Medium cut-off membrane · Health-related quality of life $\cdot$ Restless legs syndrome $\cdot$ Dialysis symptoms

\begin{abstract}
Introduction: A new generation of hemodialysis (HD) membranes called medium cut-off (MCO) membranes possesses enhanced capacities for middle molecule clearance, which have been associated with adverse outcomes in this population. These improvements could potentially positively impact patient-reported outcomes (PROs). Objective: The objective of this study was to evaluate the impact of MCO membranes on PROs in a cohort of HD patients in Colombia. Methods: This was a prospective, multicenter, observational cohort study of 992 patients from 12 renal clinics in Colombia who were switched from high-flux HD to MCO therapy and observed for 12 months. Changes in Kidney Disease Quality of Life 36-Item Short Form Survey (KDQoL-SF36) domains, Dialysis Symptom Index (DSI), and restless legs syndrome (RLS) 12 months after switching to MCO membranes were compared with time on high-flux membranes. Repeated measures of ANOVA were used to evaluate changes in KDQoL-SF36 scores; severity scoring was used to assess DSI
\end{abstract}

karger@karger.com www.karger.com/bpu

Karger $\stackrel{\text { ' }}{5}$

GOPEN ACCESS
(C) 2020 The Author(s)

Published by S. Karger AG, Basel

This article is licensed under the Creative Commons AttributionNonCommercial-NoDerivatives 4.0 International License (CC BYNC-ND) (http://www.karger.com/Services/OpenAccessLicense). Usage and distribution for commercial purposes as well as any distribution of modified material requires written permission. changes over time; Cochran's Q test was used to evaluate changes in frequency of diagnostic criteria of RLS. Results: During 12 months of follow-up, 3 of 5 KDQoL-SF36 domains improved compared with baseline: symptoms $(p<0.0001)$, effects of kidney disease $(p<0.0001)$, and burden of kidney disease $(p<0.001)$. The proportion of patients diagnosed with RLS significantly decreased from $22.1 \%$ at baseline to $10 \%$ at 12 months $(p<0.0001)$. No significant differences in the number of symptoms (DSI, $p=0.1$ ) were observed, although their severity decreased $(p=0.009)$. Conclusions: In conventional HD patients, the expanded clearance of large middle molecules with MCO-HD membranes was associated with higher health-related quality of life scores and a decrease in the prevalence of RLS. 02020 The Author(s)

Published by S. Karger AG, Basel

\section{Introduction}

Patient-reported measures (PRMs), including patient-reported outcomes (PROs), cover "any report coming from patients about a health condition and its treatment without interpretation of the patient's response by 
a clinician or anyone else" and play a critical role in medical care as a source of information about clinical experiences and outcomes for patients [1]. The Standardized Outcomes in Nephrology (SONG) initiative prioritized the key research questions most relevant to stakeholders, strongly suggesting that patients' main needs are focused on living well with chronic kidney disease (CKD) $[2,3]$.

Health-related quality of life (HRQoL) is a PRO that considers the subjective point of view of the patient and supports the evaluation of outcomes and healthcare quality [4]. Clinicians have long recognized the importance of measuring how people are feeling and the extent to which they are able to function in daily activities [5]. Patients on dialysis suffer from poor HRQoL, both from the myriad symptoms of end-stage renal disease (ESRD) and from the physical and mental burdens placed on them by their specific treatments [6].

The physical and mental HRQoL of dialysis patients (as measured by the 36-Item Short Form Survey [SF-36]) is worse than that observed for patients with chronic angina, diabetes mellitus, chronic lung disease, or hypertension [7]. In addition, dialysis patients also experience significantly higher rates of malnutrition, inflammation, hospitalization, and mortality compared with the healthy population $[8,9]$. Preserving HRQoL may have important implications; Kalantar-Zadeh and colleagues showed that each 10-unit decrease in SF-36 score was associated with a 2.07-fold increase in the relative risk of death compared with the previous 12 months [9]. In addition, Liebman and colleagues [10] published comparable data showing that a mental component score decrease of $\geq 5$ was associated with an increase in the risk of mortality. Over half of patients receiving chronic dialysis are expected to suffer from the effects of a range of symptoms, including fatigue, pain, pruritus, muscle cramps, difficulty with sleep, sexual dysfunction, and nausea, with the severity of symptoms adversely affecting patients' quality of life (QoL) in terms of physical and psychosocial functioning $[11,12]$.

The impact of contemporary renal replacement therapies on a patient's perceived HRQoL is critical to treatment success. While hemodialysis (HD) therapy removes small solutes, the removal of larger molecules $>25 \mathrm{kDa}$ (often termed large middle molecules) is limited. Hemodiafiltration (HDF) therapy can remove middle molecules more effectively than HD $[13,14]$. However, the effect of an improved uremic environment resulting from the clearance of middle molecules remains unclear based on randomized studies $[15,16]$.

Impact of MCO Membranes on PatientReported Outcomes: COREXH Registry
Advances in membrane technology have led to the development of novel medium cut-off (MCO) membranes that have enhanced selectivity and increased permeability to conventional and large middle molecules [17]. This results in a steep sieving curve in which the molecular weight retention onset and molecular weight cut-off are very close to each other while remaining lower than that of albumin, mimicking the filtration profile of the native kidney [18]. The application of these membranes in clinical dialysis is known as expanded hemodialysis ( $\mathrm{HDx}$ ) therapy $[17,19,20]$ due to the enhanced clearance of large middle molecules, which are associated with cardiovascular disease, immune modulation, and proteinenergy wasting [21]. Initial studies have demonstrated that the MCO membrane removes toxins at least as effectively as a hemofilter used in HDF mode [22]. The goal is that this enhanced removal will improve PROs and QoL for dialysis patients. Our objective was to determine the impact of the MCO membrane on outcomes, including HRQoL, presence and severity of symptoms, as well as diagnostic criteria of restless legs syndrome (RLS), in a large multicentric cohort of patients in the Expanded Hemodialysis Registry Protocol in Colombia (COREXH, ISRCTN45211359, ISRCTN45211359). https://doi.org/10.1186/

\section{Materials and Methods}

\section{Study Design and Patients}

The COREXH registry is a prospective, multicenter, observational cohort study of patients undergoing dialysis with the MCO membrane in Colombia between September 1, 2017, and November 30, 2017. Patients with CKD who had been undergoing HD therapy for more than 90 days at a Renal Therapy Services network clinic that met Association for the Advancement of Medical Instrumentation water quality standards were invited to participate. Patients were at least 18 years of age and received HD therapy for a minimum of $4 \mathrm{~h}$ thrice weekly using the MCO dialyzer (Theranova ${ }^{\circledR}$; Baxter, Deerfield, IL, USA). Patients with a life expectancy of $<6$ months or an active infection diagnosed within the previous 4 weeks were not invited to participate. Patients were prospectively followed for 12 months from enrollment. We obtained information on the baseline state of health of the patients before the prescription of therapy with the MCO dialyzer.

As this was an observational study, where the procedure of $\mathrm{HD}$ was not changed in any respect, the study was considered to be without risk. All patients provided written informed consent, and the study was conducted in accordance with the principles of the Helsinki Declaration and Good Clinical Practices. The clinical research ethics committee of Renal Therapy Services reviewed and approved the protocol (original study protocol dated July 31, 2017, version 1.0). 
Fig. 1. Patient flow for the COREXH registry. A total of 1,000 patients signed informed consent at 12 centers. From these, 8 patients did not meet participation criteria, leaving 992 patients eligible for baseline analysis. A total of 638 patients were eligible for 12-month follow-up assessment, with 354 lost to follow-up for the reasons provided. PD, peritoneal dialysis.

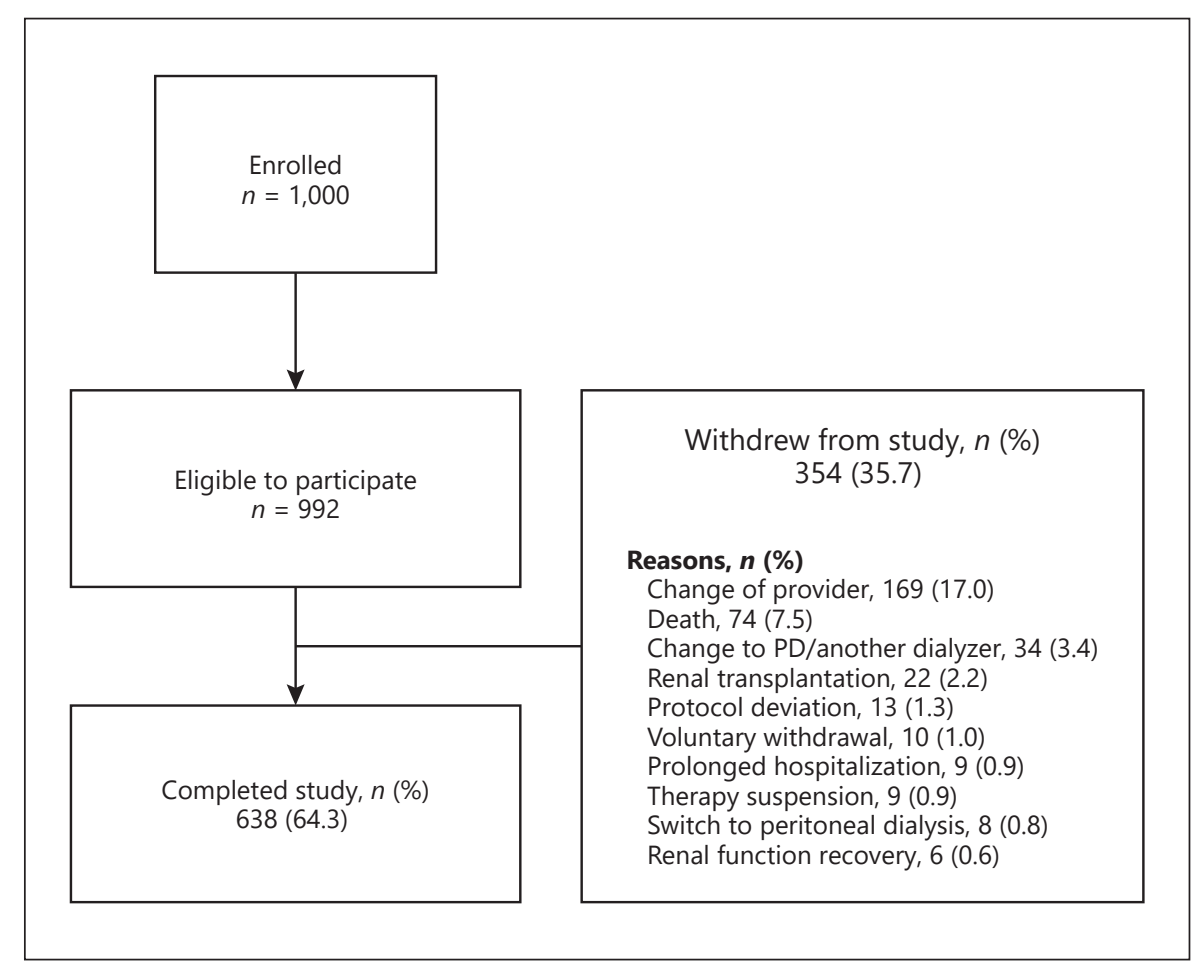

\section{Assessments}

Baseline (before switching to therapy with the MCO dialyzer) demographic and disease characteristics - including age; sex; cause of CKD; past medical history of hypertension, diabetes mellitus, and acute cardiovascular events; comorbidity index; Karnofsky performance status; and date of initiating chronic renal replacement therapy - were collected from patient electronic medical records. HD treatment parameters, including session duration, number of sessions per week, blood flow, dialysate flow, and type of vascular access, were recorded. Baseline values of KDQoL-SF36, Dialysis Symptom Index (DSI), and diagnostic criteria for RLS were captured from patient electronic medical records, and these measures were repeated at 6 and 12 months of follow-up. KDQoL-36 and DSI values were self-reported responses to surveys offered in hard copy format to the patient at the clinic with the process supported by a social worker at each site. For KDQoL-36, we used a version adapted cross-culturally into Colombian Spanish [23]. The nonresponse rate was 2.2\%; the PRO measurements were made according to convenience, either before or after the midweek dialysis session. Patients diagnosed with RLS had to meet the 4 essential criteria proposed by the International Restless Legs Syndrome Study Group (IRLSSG) published in 2003 [24]. The treating nephrologist evaluated the presence of these criteria throughout the observation period.

\section{Statistical Analysis}

A statistical description was prepared for all the variables, including calculations of the central tendency measures and dispersion for quantitative variables, and absolute frequencies and percentages for qualitative variables. Scores corresponding to each of the 5 domains of KDQoL-36 were summarized as means and stan- dard deviations (SDs). For the DSI, 2 scoring systems were considered: an overall scoring system, which was the total of all symptoms existing at the time of the evaluation, and a severity scoring system, in which each item was given a score between 1 and 5. Both scoring systems were summarized using means, medians, SDs, and interquartile ranges (IQRs). For RLS, the percentage of positive cases was calculated.

Changes in KDQoL-SF36 domains and results of DSI severity scoring over time were assessed using repeated measures ANOVA; a "one within" design was considered. To deal with lack of sphericity, $p$ values were adjusted using Box's conservative epsilon. For overall scoring of the DSI, the changeover repeated measures were evaluated using a nonparametrical approach (Friedman test). Cochran's Q test was used to statistically evaluate if the percentage of patients having RLS changed over time as this test considers dependence between dichotomic values evaluated in repeated measures. Statistical analyses were performed using STATA $14^{\circledR}$ statistics software (StataCorp LLC, TA, USA). Considering the large sample size of the study, we selected a conservative $p$ value of 0.01 for hypothesis testing.

\section{Results}

\section{Patient Demographics}

Of 1,000 patients who consented to participate, 992 from 12 clinics were included in the baseline analysis, with 638 remaining for the 12-month follow-up (Fig. 1). Most of the patients were male $(62 \%)$, with a mean age at 


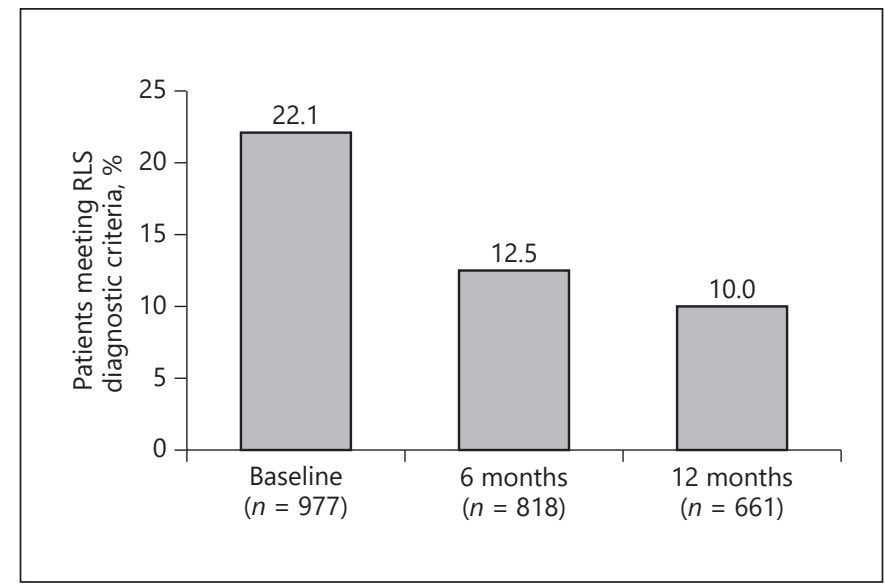

Fig. 2. Longitudinal changes in patients meeting RLS diagnosis over 12 months of follow-up. Proportion of patients meeting the RLS diagnostic criteria at baseline as well as 6 and 12 months of follow-up after switching to the medium cut-off membrane. RLS, restless legs syndrome.

enrollment of 60.5 years (SD 15.1 years). A total of 917 patients $(92.4 \%)$ had a history of hypertension, with 436 (43.9\%) and 364 (36.7\%) patients having a history of diabetes or cardiovascular disease, respectively. At the time of enrollment, included patients had been receiving highflux HD for a median of 3.7 years (IQR 6.2) (Table 1). Dialysis and laboratory parameters are presented in Table 2 .

\section{Kidney Disease Quality of Life 36-Item Short Form Survey}

Longitudinal Kidney Disease Quality of Life 36-Item Short Form Survey (KDQoL-36) scores for each of the 5 domains are shown in Table 3. Following 12 months of therapy with the MCO membrane, mean scores significantly increased from baseline for symptoms/problems (baseline: 78.6 [SD 15.8], 12 months: 81.5 [14.9]; $p<0.0001$ ), effects of kidney disease (baseline: 69.7 [22.3], 12 months: 75.1 [21.0]; $p<0.0001)$, and burden of kidney disease (baseline: 46.2 [27.5], 12 months: 50.2 [32.2]; $p<0.0001)$. Scores for the mental and physical domains remained stable and did not change significantly from baseline. We did not observe any relevant differences in KDQoL-36 domains when analyzed by participating center (see online suppl. Table 1, available at www.karger.com/doi/10.1159/000508803).
Table 1. Patient demographics and characteristics at baseline

\begin{tabular}{lcl}
\hline Baseline characteristics & $N=992$ \\
\hline Age, mean (SD), years & 60.5 & $(15.1)$ \\
Sex, $n$ (\%) & & \\
$\quad$ Male & 619 & $(62.4)$ \\
$\quad$ Female & 373 & $(37.6)$ \\
Medical history, $n(\%)$ & & \\
$\quad$ Hypertension & 917 & $(92.4)$ \\
$\quad$ Diabetes & 436 & $(43.9)$ \\
$\quad$ Cardiovascular disease & 364 & $(36.7)$ \\
Comorbidity index, $n$ (\%) & & \\
$\quad$ 0 to 3 & 802 & $(80.9)$ \\
$\quad 4$ to 6 & 170 & $(17.1)$ \\
$\quad \geq 7$ & 20 & $(2)$ \\
Karnofsky PS score, ${ }^{\text {a }}$ median (IQR) & 80.0 & $(70,90)$ \\
Dialysis vintage, median (IQR), years & 3.7 & $(1.6,7.8)$ \\
\hline
\end{tabular}

$\mathrm{IQR}$, interquartile range; $\mathrm{PS}$, performance status; $\mathrm{SD}$, standard deviation. ${ }^{\text {a }}$ Data available for 914 patients.

\section{Restless Legs Syndrome Diagnostic Criteria}

The proportion of patients meeting RLS diagnostic criteria significantly decreased over the follow-up period (Fig. 2), with 216/977 (22.1\%), 102/818 (12.5\%), and $66 / 661(10.0 \%)$ of patients meeting the criteria at baseline and 6 and 12 months, respectively $(p<0.0001)$. A post hoc comparison showed statistically significant differences between each pair of repeated observations (baseline vs. 6 months: $p<0.0001$; baseline vs. 12 months: $p<0.0001$; 6 vs. 12 months: $p=0.003$ ).

\section{Dialysis Symptom Index}

No significant differences in the mean number of symptoms were reported at baseline compared with 6 or 12 months of follow-up, being 10.3, 10.3, and 10.0, respectively $(p=0.1)$. However, mean severity scores significantly decreased from 30.7 (SD 22.3) at baseline to 29.9 (32) at 6 months and 28.5 (21.7) at 12 months of follow-up ( $p=0.009$ ) (Table 4). All 30 DSI items were reported at a lower frequency at 6 and 12 months compared with baseline, with marginally significant reductions in shortness of breath, dizziness/lightheadedness, and difficulty falling asleep observed. Four items consistently had the highest scores over time (dry skin, feeling tired, trouble falling asleep, and trouble staying asleep), and 4 items consistently had low scores over time (chest pain, diarrhea, swelling in legs, and vomiting) (Table 5). 
Table 2. Dialysis and laboratory parameters at baseline and 12 months of follow-up

\begin{tabular}{|c|c|c|}
\hline & Baseline, $n=992$ & 12 months, $n=638$ \\
\hline \multicolumn{3}{|l|}{ Dialysis parameters } \\
\hline \multicolumn{3}{|l|}{ Vascular access, $\%(n)$} \\
\hline AV fistula & $83.06(824)$ & $83.54(533)$ \\
\hline Catheter & $14.52(144)$ & $13.95(89)$ \\
\hline Graft & $2.42(24)$ & $2.51(16)$ \\
\hline \multicolumn{3}{|l|}{ Treatments/week, \% ( $n)$} \\
\hline 3 & $99.19(984)$ & $99.06(632)$ \\
\hline 4 & $0.71(7)$ & $0.94(6)$ \\
\hline 5 & $0.10(1)$ & - \\
\hline Duration of a dialysis session, mean (SD), h & $4.01(0.11)$ & $4.01(0.11)$ \\
\hline Dialysate flow, mean (SD), $\mathrm{mL} / \mathrm{min}$ & $486.99(52.21)$ & $481.50(54.56)$ \\
\hline Blood flow, mean (SD), $\mathrm{mL} / \mathrm{min}$ & $352.05(51.31)$ & $355.43(49.97)$ \\
\hline \multicolumn{3}{|l|}{ Dialyzer type, \% $(n)$} \\
\hline Dicea $110 \mathrm{G}\left(1.1 \mathrm{~m}^{2}\right)$ & $0.20(2)$ & - \\
\hline Xenium XPH-210 $\left(2.1 \mathrm{~m}^{2}\right)$ & $0.40(4)$ & - \\
\hline Polyflux $140 \mathrm{H}\left(1.4 \mathrm{~m}^{2}\right)$ & $2.22(22)$ & - \\
\hline Revaclear $300\left(1.6 \mathrm{~m}^{2}\right)$ & $78.02(774)$ & - \\
\hline Revaclear $400\left(1.8 \mathrm{~m}^{2}\right)$ & $19.15(190)$ & - \\
\hline Theranova $400\left(1.7 \mathrm{~m}^{2}\right)$ & - & $91.22(582)$ \\
\hline Theranova $500\left(2.0 \mathrm{~m}^{2}\right)$ & - & $8.78(56)$ \\
\hline \multicolumn{3}{|l|}{ Clinical laboratory parameters } \\
\hline Albumin, mean (SD), g/dL & $4.04(0.33)$ & $3.98(0.34)$ \\
\hline Hemoglobin, mean (SD), g/dL & $11.89(1.72)$ & $11.87(1.66)$ \\
\hline Phosphorous, mean (SD), mg/dL & $4.60(1.38)$ & $4.54(1.32)$ \\
\hline PTHi, median (IQR), pg/mL & $327.80(165.30,625.30)$ & $309.50(173.00,562.00)$ \\
\hline hsCRP, mean (SD), mg/L & $1.11(2.83)$ & $2.36(15.68)$ \\
\hline $\mathrm{spKt} / V_{\text {urea }}$, mean $(\mathrm{SD})$ & $1.62(0.34)$ & $1.71(0.36)$ \\
\hline
\end{tabular}

$\mathrm{AV}$, arteriovenous; hsCRP, high-sensitivity C-reactive protein; IQR, interquartile range; PTHi, parathyroid hormone; $\mathrm{SD}$, standard deviation; spKt/ $V_{\text {urea }}$, standardized Kt/ $V_{\text {urea }}$.

Table 3. Changes in KDQoL-36 score over 12 months of follow-up

\begin{tabular}{lllllc}
\hline KDQoL-36 domain & Statistic & $\begin{array}{l}\text { Baseline, } \\
n=971\end{array}$ & $\begin{array}{l}\text { 6 months, } \\
n=808\end{array}$ & $\begin{array}{l}12 \text { months, } \\
n=642\end{array}$ & $p$ value $^{\mathrm{a}}$ \\
\hline Symptoms/problems & Mean & 78.6 & 81.0 & 81.5 & $<0.0001$ \\
& SD & 15.8 & 15.4 & 14.9 & $<0.0001$ \\
\hline Effects of kidney disease & Mean & 69.7 & 72.8 & 75.1 & 21.0 \\
\hline Burden of kidney disease & SD & 22.3 & 22.0 & 50.2 & 0.3 \\
\hline SF-12 physical & Mean & 46.2 & 48.9 & 32.3 & 41.7 \\
\hline SF-mental & SD & 27.5 & 29.9 & 10.5 & 0.01 \\
\hline
\end{tabular}

KDQoL-36, Kidney Disease Quality of Life 36-Item Short Form Survey; SD, standard deviation; SF, short form. ${ }^{a}$ For hypothesis testing, type-I error significance was set at $p=0.01$. 
Table 4. Changes in DSI over 12 months of follow-up

\begin{tabular}{llllll}
\hline DSI domain & Statistic & \multicolumn{3}{c}{ Baseline, 6 months, } & \multicolumn{2}{c}{12 months, $p$} \\
& & $n=977$ & $n=813$ & $n=642$ & value \\
\hline Number of & Mean & 10.3 & 10.3 & 10.0 & $0.1^{\mathrm{a}}$ \\
symptoms & SD & 6.6 & 6.7 & 6.6 & \\
& Median & 9 & 9 & 9 & $\mathrm{NA}$ \\
& IQR & 10 & 10 & 9 & \\
\hline Symptom & Mean & 30.7 & 29.9 & 28.5 & $0.009^{\mathrm{b}}$ \\
severity score & SD & 22.3 & 32.0 & 21.7 & \\
& Median & 26 & 26 & 23 & $\mathrm{NA}$ \\
& IQR & 32 & 30 & 31 & \\
\hline
\end{tabular}

DSI, Dialysis Symptom Index; IQR, interquartile range; NA, not applicable; SD, standard deviation. ${ }^{a}$ By Friedman's test. ${ }^{b}$ By ANOVA.

\section{Discussion/Conclusion}

Chronic HD aims to improve survival while maintaining or minimizing any negative impact on patient QoL. High-flux membranes are unable to effectively remove large middle molecules or those molecules that have a molecular diameter larger than the pore size, which may contribute to poor nutritional and inflammation status and reduced QoL [25]. The MCO membrane enables expanded removal of large middle molecules compared with high-flux HD, including myoglobin, $\beta 2$ microglobulin, macroglobulin, homocysteine, as well as $\lambda$ - and $\kappa$-free light chains [22]. This property could translate into myriad benefits for patients receiving dialysis $[22,26]$.

Table 5. Changes in frequency of DSI over 12 months of follow-up

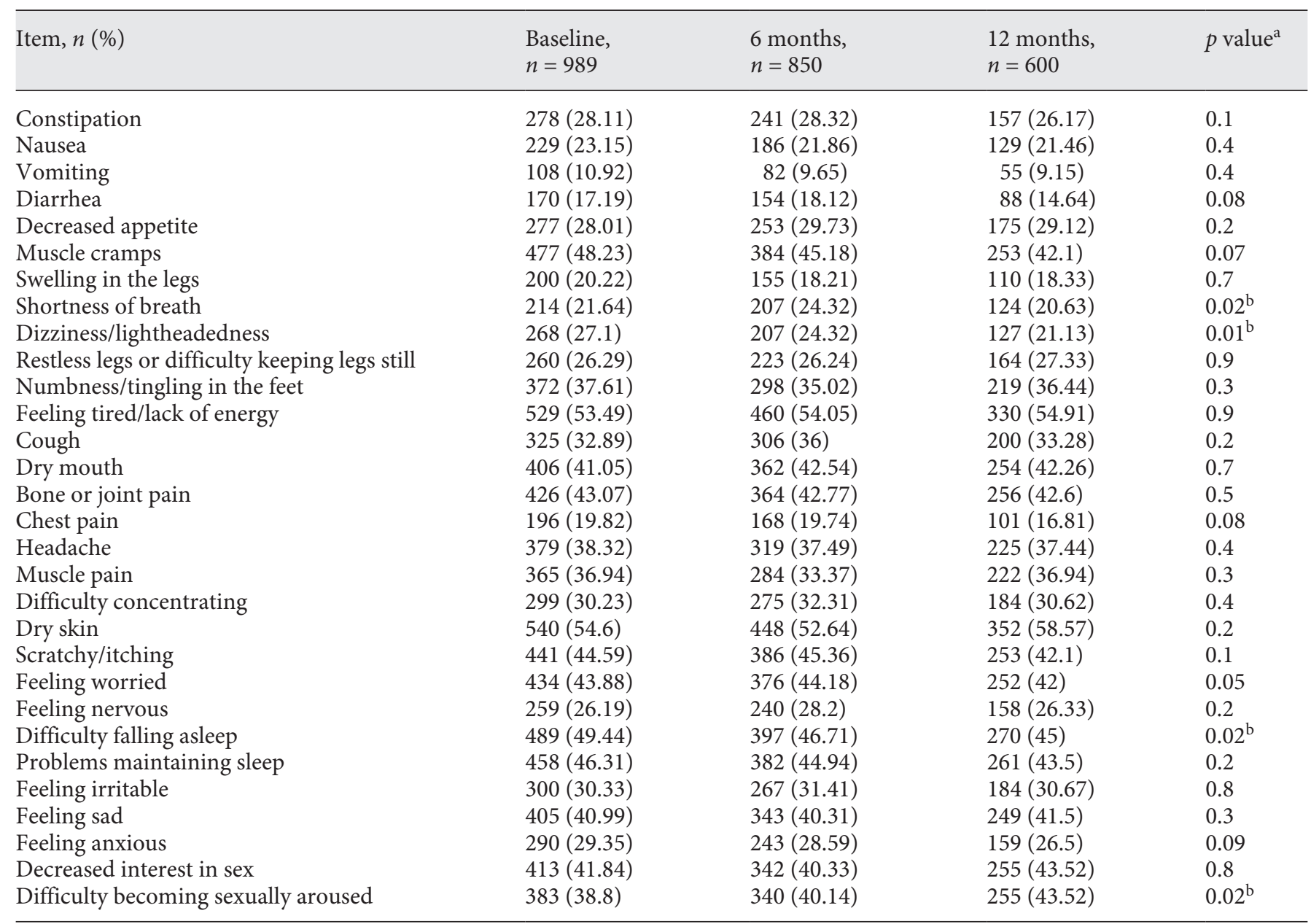

DSI, Dialysis Symptom Index. ${ }^{a}$ Cochran's $Q$ test for proportions at moments in time. ${ }^{\mathrm{b}}$ Considered to be marginally significant.

Impact of MCO Membranes on PatientReported Outcomes: COREXH Registry 
Improving the HRQoL and PROs for dialysis patients is a therapeutic goal, with studies demonstrating the impact of HRQoL on patient outcomes [27]. Poor mental health is an independent risk factor for mortality [28], and decreases in the 2 main QoL domains mental and physical health - correlate with reduced PROs and survival $[29,30]$. Finally, QoL correlates with nutrition, potentially predicting longevity [31]. Rambod and colleagues [31] reported that the MalnutritionInflammation Score (MIS) correlated with levels of middle molecules, including interleukin-6 and C-reactive protein, with a higher MIS being associated with lower SF-36 scores and ultimately mortality in patients receiving HD over 5 years. These results match ours, supporting the hypothesis that expanded clearance of large middle molecules improves measures of QoL over 12 months.

Using KDQoL-36, the Dialysis Outcomes and Practice Patterns Study demonstrated that the adjusted mortality risk increased by $25 \%$ with each 10 -point reduction in the physical component summary, $13 \%$ with each 10 -point reduction in the mental component summary, and $11 \%$ with each 10-point reduction in the kidney disease component summary [32]. Similarly, the CONTRAST study demonstrated that decreases in physical function, emotional health, and social functioning were significantly associated with mortality over 2 years [33] and were independent of age [33]. It is encouraging that we observed a positive impact of the expanded removal of large middle molecules on QoL measures in adult patients.

To our knowledge, this is the first study that measures HRQoL and the frequency and severity of symptoms, as well as the presence of diagnostic criteria for RLS, in patients receiving MCO membrane-based HD. Significant improvements were observed in 3 of the 5 HRQoL domains measured by KDQoL-36 - symptoms/problems, effects of kidney disease, and burden of kidney disease - and were most pronounced in the kidney disease effects domain. The effect size is modest but consistent across the full 12-month follow-up period, suggesting that the expanded clearance of large middle molecules provided by the MCO membrane may be associated with improvements in patient QoL. Regarding the size of this effect, the difference in QoL found was between 3 and 5 points per domain, which is slightly higher than that found between different types of dialysis therapy in the USA [34]; furthermore, and contrary to the expected outcomes for patients receiving chronic dialysis, QoL trended towards improvement over the course of the follow-up.
Approximately 2.5 to $15 \%$ of the healthy population is estimated to meet the International Diagnostic Criteria for RLS. For patients with ESRD, 20 to $25 \%$ of patients show overt (moderate-to-severe) RLS, while 62\% present milder forms $[35,36]$. While the pathogenic mechanisms for uremic RLS are unknown, the most widely accepted hypothesis for idiopathic RLS is a dysfunction of the dopaminergic system and reduced iron stores in specific regions in the brain [37]. A removal rate of $35 \%$ of $\alpha 1$-microglobulin ( $\alpha 1-\mathrm{MG}$; a heme scavenger) is associated with an improvement in RLS symptoms [38]; it is not clear if elevated a $1-\mathrm{MG}$ levels are toxic per se and, for now, should be used as a biomarker [38]. It is noteworthy that patients can completely recover from uremic RLS symptoms 1-21 days after a successful kidney transplant [39]. In addition, for patients who again become dependent on dialysis, RLS symptoms return 10-60 days after reinitiating HD, supporting the role of uremic toxicity in the pathogenesis of RLS [39]. This is likely to be due to the levels of middle molecules that were not well removed during dialysis either prior to transplant or after rejection. Importantly, we show a significant $(54.86 \%)$ decrease in the proportion of patients meeting the diagnostic criteria for RLS at 12 months compared with baseline. Combined with the difficulties in correlating uremic toxin removal with RLS occurrence as well as the consistent, yet limited, data indicating HD has minimal impact on RLS [40], our results suggest that the expanded clearance of large middle molecules with the MCO membrane (comparable with the natural kidney) may alleviate the development and impact of RLS. In addition, we observed a modest reduction in the severity of dialysis symptoms (mean DSI) after switching to the MCO membrane. This observation may contribute to our understanding of the importance of middle molecule removal on common dialysis symptoms including anorexia, fatigue, or pruritus. Of note is the decrease in the proportion of patients with difficulties falling asleep, as well as in the presence of dizziness/lightheadedness, as this was likely related to improvements in RLS and sleep pattern, which has been previously linked to the clearance of middle molecules in the literature $[25,41]$.

Our study has several strengths supporting the validity of our results. We observed patients prospectively for 12 months, enabling the longitudinal measurement of outcomes, including QoL and PROs, and comparison with historical baseline values, allowing us to directly assess the impact of switching to the MCO membrane. Our data were obtained from a large cohort of patients $(N=$ 
992) from multiple clinics, with every attempt made to maintain the quality of care. Thus, we can be confident that our observations provide a robust measure of current treatment practices and their impacts. Our study does have limitations. Being a registry, there is no randomization or blinding to the intervention, creating potential for biases in the observed and the observer; in addition, there was no comparator group, and this may decrease to some extent the level of causality that can be attributed to the different practices. However, the substantial number of patients and the longitudinal follow-up period, in our view, make this a nonnegligible body of evidence.

After switching to the MCO membrane, the population in this study presented not only a statistically significant improvement in their HRQoL (measured with KDQoL-36) in 3 of the 5 domains (symptoms/problems, effects of kidney disease, and burden of kidney disease) but also a significant decrease in the prevalence of RLS. If validated in other studies, these findings may support the routine use of MCO membranes in HD patients, enhancing the standards of care.

\section{Acknowledgements}

The authors wish to express their gratitude to all the patients and nursing teams who participated in the study. The authors thank all the investigators who participated in the Colombian Registry of Expanded Hemodialysis study: Alexander Arias, MD (Medellín), Adolfo E. Capella, MD (Armenia), Carlos Y. Coronado, MD (Ibagué), Leyder Corzo, MD (Bogotá), Maria P. Dazzarola, MD (Cali), Daniel A. Ducuara, MD (Bogotá), Rafael A. Gómez, MD (Cali), Mario D. Munevar, MD (Barranquilla), Edward A. Martinez, MD (Bucaramanga), Anthony E. Martinez, MD (Bogotá), Alejandra P. Molano, MD (Bogotá), and Sylvia C. Quiñonez, MD (Bogotá). All investigators are employees of RTS Ltd. Colombia, an affiliate of Baxter Healthcare.

\section{Statement of Ethics}

All patients provided written informed consent, and the study was conducted in accordance with the principles of the Helsinki Declaration and Good Clinical Practices. The clinical research ethics committee of Renal Therapy Services reviewed and approved the protocol (original study protocol dated July 31, 2017, version 1.0).

\section{Conflict of Interest Statement}

Drs. Alarcon and Bunch and Mr. Sanabria are full-time employees of Renal Therapy Services-Latin America, Bogotá, Colombia. Drs. Ardila and Zuñiga and Ms. Vesga are full-time employees of Renal Therapy Services-Colombia, Bogotá, Colombia. Dr. Rivera is a full-time employee of Baxter Healthcare Corporation, Chicago, IL, USA, and has a patent for Membran zur Behandlung des Rest Leg Syndrome pending. Mr. Sánchez has received an honorarium for consultancy from Renal Therapy Services-Colombia.

\section{Funding Sources}

This study was supported by Renal Therapy Services-Colombia, an independent entity owned by Baxter International, Inc. Funding for this study and to support the preparation of the manuscript was provided by Baxter Healthcare Corporation, Deerfield, IL, USA. Editorial support for the development of the manuscript was provided by Daniel Johnson, $\mathrm{PhD}$, Apurva Davé, $\mathrm{PhD}$, and Nicholas Schumacker, PhD, SciMentum (Nucleus Global), with funding provided by Baxter Healthcare Corporation.

\section{Author Contributions}

J.C.A., A.B., F.A., and E.Z.: original research project conception and design, data acquisition, and data interpretation. J.V. and M.S.: original research project conception and design, data acquisition, statistical analysis, and data interpretation. A.R.: original research project conception and design and data interpretation. R.S.: original research project conception and design, statistical analysis, and data interpretation. All authors have been involved in the drafting of the manuscript or revising it critically for important intellectual content and provided final approval of the version to be published. All authors verify that they have met all the journal's requirements for authorship. All authors agree to be accountable for all aspects of the work, ensuring the accuracy and integrity of the publication. All authors approved the final manuscript draft submitted for publication. The authors received no financial compensation for the development of the manuscript.

\section{References}

1 Peipert JD, Hays RD. Methodological considerations in using patient reported measures in dialysis clinics. J Patient Rep Outcomes. 2017; $1(1): 11$.

2 Tong A, Crowe S, Chando S, Cass A, Chadban SJ, Chapman JR, et al. Research priorities in CKD: report of a national workshop conducted in Australia. Am J Kidney Dis. 2015;66(2): 212-22.

Impact of MCO Membranes on PatientReported Outcomes: COREXH Registry
3 Tong A, Manns B, Hemmelgarn B, Wheeler DC, Tugwell P, Winkelmayer WC, et al. Standardised Outcomes in Nephrology - Haemodialysis (SONG-HD): study protocol for establishing a core outcome set in haemodialysis. Trials. 2015;16:364.

4 Gibbons E, Fitzpatrick R. A structured review of patient-reported outcome measures for people with chronic kidney dis- ease. 2010 https://phi.uhce.ox.ac.uk/pdf/ PROMS_Oxford_KidneyReview 24112010.pdf.

5 Guyatt GH, Ferrans CE, Halyard MY, Revicki DA, Symonds TL, Varricchio CG, et al. Exploration of the value of health-related qualityof-life information from clinical research and into clinical practice. Mayo Clin Proc. 2007; 82(10):1229-39. 
6 Jaar BG, Chang A, Plantinga L. Can we improve quality of life of patients on dialysis? Clin J Am Soc Nephrol. 2013;8(1):1-4.

7 Mittal SK, Ahern L, Flaster E, Maesaka JK, Fishbane S. Self-assessed physical and mental function of haemodialysis patients. Nephrol Dial Transplant. 2001;16(7):1387-94.

8 Ikizler TA, Cano NJ, Franch H, Fouque D, Himmelfarb J, Kalantar-Zadeh K, et al. Prevention and treatment of protein energy wasting in chronic kidney disease patients: a consensus statement by the International Society of Renal Nutrition and Metabolism. Kidney Int. 2013;84(6):1096-107.

9 Kalantar-Zadeh K, Kopple JD, Block G, Humphreys MH. Association among SF36 quality of life measures and nutrition, hospitalization, and mortality in hemodialysis. J Am Soc Nephrol. 2001;12(12):2797-806.

10 Liebman S, Li NC, Lacson E. Change in quality of life and one-year mortality risk in maintenance dialysis patients. Qual Life Res. 2016; 25(9):2295-306.

11 Merkus MP, Jager KJ, Dekker FW, de Haan RJ, Boeschoten EW, Krediet RT. Physical symptoms and quality of life in patients on chronic dialysis: results of The Netherlands Cooperative Study on Adequacy of Dialysis (NECOSAD). Nephrol Dial Transplant. 1999; 14(5):1163-70.

12 Abdel-Kader K, Unruh ML, Weisbord SD. Symptom burden, depression, and quality of life in chronic and end-stage kidney disease. Clin J Am Soc Nephrol. 2009;4(6):1057-64.

13 Blankestijn PJ, Ledebo I, Canaud B. Hemodiafiltration: clinical evidence and remaining questions. Kidney Int. 2010;77(7):581-7.

14 Grooteman MP, van den Dorpel MA, Bots ML, Penne EL, van der Weerd NC, Mazairac $\mathrm{AH}$, et al. Effect of online hemodiafiltration on all-cause mortality and cardiovascular outcomes. J Am Soc Nephrol. 2012;23(6): 1087-96.

15 Mazairac AH, de Wit GA, Grooteman MP, Penne EL, van der Weerd NC, den Hoedt CH, et al. Effect of hemodiafiltration on quality of life over time. Clin J Am Soc Nephrol. 2013; 8(1):82-9.

16 Ward RA, Schmidt B, Hullin J, Hillebrand GF, Samtleben W. A comparison of on-line hemodiafiltration and high-flux hemodialysis: a prospective clinical study. J Am Soc Nephrol. 2000;11(12):2344-50.
17 Ronco C, Marchionna N, Brendolan A, Neri M, Lorenzin A, Martínez Rueda AJ. Expanded haemodialysis: from operational mechanism to clinical results. Nephrol Dial Transplant. 2018;33(Suppl 3):iii41-47.

18 Boschetti-de-Fierro A, Voigt M, Storr M, Krause B. MCO membranes: enhanced selectivity in high-flux class. Sci Rep. 2015;5: 18448.

19 Ronco C. The rise of expanded hemodialysis. Blood Purif. 2017;44(2):I-VIII. http://dx.doi. org/10.1159/000476012.

20 Belmouaz M, Bauwens M, Hauet T, Bossard V, Jamet P, Joly F, et al. Comparison of the removal of uraemic toxins with medium cutoff and high-flux dialysers: a randomized clinical trial. Nephrol Dial Transplant. 2020; 35(2): 328-335. http://dx.doi.org/10.1093/ ndt/gfz189.

21 Wolley M, Jardine M, Hutchison CA. Exploring the clinical relevance of providing increased removal of large middle molecules. Clin J Am Soc Nephrol. 2018;13(5):805-14.

22 Kirsch AH, Lyko R, Nilsson LG, Beck W, Amdahl M, Lechner P, et al. Performance of hemodialysis with novel medium cut-off dialyzers. Nephrol Dial Transplant. 2017;32(1): 165-72.

23 Chavez K, Duarte A, Vega J. Adaptación transcultural del cuestionario KDQOL SF 36 para evaluar calidad de vida en pacientes con enfermedad renal crónica en Colombia. Revista Med. 2013;21(2):12-7.

24 Allen RP, Picchietti D, Hening WA, Trenkwalder C, Walters AS, Montplaisi J, et al. Restless legs syndrome: diagnostic criteria, special considerations, and epidemiology. A report from the restless legs syndrome diagnosis and epidemiology workshop at the National Institutes of Health. Sleep Med. 2003;4(2):101-19.

25 Florens N, Juillard L. Expanded haemodialysis: news from the field. Nephrol Dial Transplant. 2018;33(Suppl 3):iii48-52.

26 Hutchison CA, Wolley M. The rationale for expanded hemodialysis therapy (HDx). Contrib Nephrol. 2017;191:142-52.

27 Kalantar-Zadeh K, Unruh M. Health related quality of life in patients with chronic kidney disease. Int Urol Nephrol. 2005;37(2):367-78.

28 Kimmel PL, Peterson RA, Weihs KL, Simmens SJ, Boyle DH, Cruz I, et al. Aspects of quality of life in hemodialysis patients. J Am Soc Nephrol. 1995;6(5):1418-26.

29 Valdés C, García-Mendoza M, Rebollo P, Ortega T, Ortega F. Mental health at the third month of haemodialysis as a predictor of short-term survival. Nephrol Dial Transplant. 2006;21(11):3223-30.
30 Tsai YC, Hung CC, Hwang SJ, Wang SL, Hsiao SM, Lin MY, et al. Quality of life predicts risks of end-stage renal disease and mortality in patients with chronic kidney disease. Nephrol Dial Transplant. 2010;25(5):1621-6.

31 Rambod M, Bross R, Zitterkoph J, Benner D, Pithia J, Colman S, et al. Association of Malnutrition-Inflammation Score with quality of life and mortality in hemodialysis patients: a 5 -year prospective cohort study. Am J Kidney Dis. 2009;53(2):298-309.

32 Mapes DL, Lopes AA, Satayathum S, McCullough KP, Goodkin DA, Locatelli F, et al. Health-related quality of life as a predictor of mortality and hospitalization: the Dialysis Outcomes and Practice Patterns Study (DOPPS). Kidney Int. 2003;64(1):339-49.

33 van Loon IN, Bots ML, Boereboom FTJ, Grooteman MPC, Blankestijn PJ, van den Dorpel MA, et al. Quality of life as indicator of poor outcome in hemodialysis: relation with mortality in different age groups. BMC Nephrol. 2017;18(1):217.

34 Cohen DE, Lee A, Sibbel S, Benner D, Brunelli SM, Tentori F. Use of the KDQOL-36TM for assessment of health-related quality of life among dialysis patients in the United States. BMC Nephrol. 2019;20(1):112. http://dx.doi. org/10.1186/s12882-019-1295-0.

35 Zucconi M, Ferini-Strambi L. Epidemiology and clinical findings of restless legs syndrome. Sleep Med. 2004;5(3):293-9.

36 Earley CJ. Clinical practice. Restless legs syndrome. N Engl J Med. 2003;348(21):2103-9.

37 Novak M, Mendelssohn D, Shapiro CM, Mucsi I. Diagnosis and management of sleep apnea syndrome and restless legs syndrome in dialysis patients. Semin Dial. 2006;19(3):2106.

38 Sakurai K. Biomarkers for evaluation of clinical outcomes of hemodiafiltration. Blood Purif. 2013;35(Suppl 1):64-8.

39 Winkelmann J, Stautner A, Samtleben W, Trenkwalder C. Long-term course of restless legs syndrome in dialysis patients after kidney transplantation. Mov Disord. 2002;17(5): 1072-6.

40 Lerma EV. Are restless legs syndrome, pruritus or hiccoughs surrogates for inadequate dialysis? Semin Dial. 2011;24(4):406-7.

41 Scherer JS, Combs SA, Brennan F. Sleep disorders, restless legs syndrome, and uremic pruritus: diagnosis and treatment of common symptoms in dialysis patients. Am J Kidney Dis. 2017;69(1):117-28. 